Zbigniew Jelonek · Wojciech Kucharz

\title{
On smooth hypersurfaces containing a given subvariety
}

Received: 11 April 2013 / Revised: 20 June 2014

Published online: 13 January 2016

Abstract. We show when a nonsingular closed subvariety $Y$ of a nonsingular affine real variety $X$ is contained in a nonsingular hypersurface. We also solve this problem in a holomorphic case.

\section{Introduction}

Let $X^{n}$ be a nonsingular affine variety (of dimension $n$ ) over an algebraically closed field $k$. It was proved in [4] (see also [7]), that any closed nonsingular subvariety $Y^{r} \subset X^{n}$ with $n \geq 2 r+1$ is contained in a nonsingular hypersurface. Recall that a subvariety $H \subset X$ is a hypersurface if its ideal $I(H) \subset k[X]$ is generated by a single polynomial. Our aim is to prove similar results in the real algebraic case and in the complex analytic case.

Let $X$ be a real algebraic variety ( as in [1]). We say that a subvariety $H$ of $X$ is a hypersurface if the ideal

$$
I(H)=\left\{f \in \mathcal{R}(X) \mid f_{\mid H}=0\right\}
$$

is principal. Here $\mathcal{R}(X)$ denotes the ring of regular functions on $X$. We show:

Theorem 1.1. Let $X^{n}$ be a nonsingular real affine variety and let $Y^{r}$ be a closed nonsingular subvariety of $X$. Assume that either

(i) $2 r+1 \leq n$, or

(ii) $2 r=n$ and $Y$ has no compact connected components.

Then there exists a nonsingular hypersurface $H$ in $X$ with $Y \subset H$.

If $X=\mathbb{R}^{n}$, point (ii) can be extended:

The authors were partially supported by the Grant of Narodowe Centrum Nauki, Number 2013/09/B/ST1/04162.

Z. Jelonek ( $\varangle$ ): Instytut Matematyczny PAN, ul. Śniadeckich 8, 00-956 Warszawa, Poland. e-mail: najelone@cyf-kr.edu.pl

W. Kucharz: Instytut Matematyki UJ, ul. Łojasiewicza 6, 30-348 Kraków, Poland. e-mail: Wojciech.Kucharz@im.uj.edu.pl

Mathematics Subject Classification 14 R 10 - 14 R 99 
Theorem 1.2. Let $Y^{r}$ be a compact nonsingular subvariety of $\mathbb{R}^{2 r}$. If $Y$ is orientable (as $\mathcal{C}^{\infty}$ manifold), then there exists a nonsingular hypersurface $H$ in $\mathbb{R}^{2 r}$ with $Y \subset H$.

We also have the following holomorphic version of Theorem 1.1:

Theorem 1.3. Let $X^{n}$ be a Stein manifold and let $Y^{r}$ be a closed submanifold of $X$. If $(3 r+1) / 2 \leq n$, then there exists a nonsingular hypersurface $H$ in $X$ with $Y \subset H$.

By definition, in the complex analytic case, $H$ is a hypersurface in $X$, if the ideal of all holomorphic functions on $X$ vanishing on $H$ is principal.

\section{Real case}

In this section we work with affine real varieties (as in [1]). For the sake of completeness, we record the following fact.

Lemma 2.1. Let $Y \subset X$ be nonsingular affine real varieties and let $s, g_{1}, \ldots, g_{r}$ be regular functions on $X$ such that $d_{y} s \neq 0$ for every $y \in Y$ and $I(Y)=\left(g_{1}, \ldots, g_{r}\right)$. Then the hypersurface $V\left(s+\sum_{i=1}^{r} \beta_{i} g_{i}^{2}\right)$ in $X$ is nonsingular for generic $\beta_{i} \in \mathbb{R}$.

Proof. Note that $0 \in \mathbb{R}$ is a regular value of the regular function

$$
X \times \mathbb{R}^{r} \ni\left(x,\left(\beta_{1}, \ldots, \beta_{r}\right)\right) \mapsto s(x)+\sum_{i=1}^{r} \beta_{i} g_{i}(x)^{2} \in \mathbb{R} .
$$

Hence the assertion follows from the parametric transversality theorem (see [5], p. 68).

We make use of Lemma 2.1 in the following:

Theorem 2.2. Let $Y \subset X$ be nonsingular real affine varieties. Then $Y$ is contained in some nonsingular hypersurface $V(f) \subset X$ if and only if the normal bundle of $Y$ contains a one-dimensional trivial summand i.e.,

$$
\mathbf{N}_{X / Y}=\mathbf{T} \oplus \mathbf{E}^{1}
$$

where $\mathbf{E}^{1}$ denotes a trivial line bundle.

Proof. Assume that there is a nonsingular hypersurface $H=V(f) \subset X$ which contains $Y$. We have

$$
T Y \subset T H \subset T X
$$

and hence

$$
\mathbf{N}_{X / Y}=\left.\mathbf{N}_{H / Z} \oplus \mathbf{N}_{X / H}\right|_{Y}
$$

However, the normal bundle of the nonsingular hypersurface $H=V(f)$ is trivial (in fact the class of $f$ is a generator of the conormal bundle of $H$ ). 
Conversely, assume that

$$
\mathbf{N}_{X / Y}=\mathbf{T} \oplus \mathbf{E}^{1}
$$

Hence also

$$
\mathbf{N}_{X / Y}^{*}=\mathbf{T}^{*} \oplus \mathbf{E}^{1}
$$

This means that the conormal bundle $\mathbf{N}_{X / Y}^{*}$ has a nowhere vanishing section $\mathbf{s} \in$ $\Gamma\left(Y, \mathbf{N}_{X / Y}^{*}\right)$. But $\Gamma\left(Y, \mathbf{N}_{X / Y}^{*}\right)=I(Y) / I(Y)^{2}$, where $I(Y) \subset \mathcal{R}(X)$ denotes the ideal of the subvariety $Y$. Hence $\mathbf{s}$ corresponds to a regular function $s \in I(X)$ such that the class of $s$ is $\mathbf{s}$. Take a point $a \in Y$ and a system of local coordinates $\left(u_{1}, \ldots, u_{n}\right)$ at $a$ such that $Y$ is described by the local equations $u_{1}=0, \ldots, u_{t}=0$ $(t=\operatorname{codim} Y)$ near $a$. Since $\mathbf{u}_{1}, \ldots, \mathbf{u}_{t}$ freely generate the bundle $\mathbf{N}_{X / Y}^{*}$ near the point $a$, we have

$$
\mathbf{s}=\sum_{i=1}^{t} \alpha_{i} \mathbf{u}_{i},
$$

where $\alpha_{i} \in \mathcal{R}\left(U_{a}\right)$ ( $U_{a}$ denotes some Zariski open neighborhood of $a$ in $Y$ ). Since the section $\mathbf{s}$ nowhere vanishes, the $\alpha_{i}$ do not vanish simultaneously at any point of $U_{a}$. Let us compute the derivative $d_{y} s$ at the point $y \in U_{a}$.

After shrinking $X$ and $Y$ we can assume that (1) holds in $Y$. Moreover we can assume that all $\alpha_{i}$ are defined on $X$. We have

$$
s=\sum_{i=1}^{t} \alpha_{i} u_{i} \bmod I(Y)^{2},
$$

hence there are regular functions $f_{j}, h_{j} \in I(Y), j=1, \ldots, m$, such that

$$
s=\sum_{i=1}^{t} \alpha_{i} u_{i}+\sum_{j=1}^{m} f_{j} h_{j} .
$$

Now we easily see that for $y \in Y$ we have

$$
d_{y} s=\sum_{i=1}^{t} \alpha_{i} d_{y} u_{i}
$$

Since $d_{y} u_{i}, i=1, \ldots, n$, are linearly independent and not all $\alpha_{i}$ vanish at $y$, we have $d_{y} s \neq 0$. Let $I(Y)=\left(g_{1}, \ldots, g_{r}\right)$. By Lemma 2.1, the hypersurface $V\left(s+\sum_{i=1}^{r} \beta_{i} g_{i}^{2}\right)$ is nonsingular for generic $\beta_{i} \in \mathbb{R}$. Hence we can take $f=$ $s+\sum_{i=1}^{r} \beta_{i} g_{i}^{2}$.

In the sequel we need the following:

Lemma 2.3. Let $X$ be a nonsingular real affine variety and let $\mathbf{F}$ be a real algebraic vector bundle on $X$. If rank $\mathbf{F}>\operatorname{dim} \mathbf{X}$, then $\mathbf{F}$ has a one-dimensional trivial summand i.e.,

$$
\mathbf{F}=\mathbf{T} \oplus \mathbf{E}^{1}
$$


Proof. The category of algebraic real vector bundles on $X$ is equivalent with the category of finitely generated projective $\mathcal{R}(X)$-modules ([1], p. 305). Hence Lemma 2.3 is a special case of Serre's splitting theorem (see [10]).

Lemma 2.4. Let $X$ be a nonsingular real affine variety and let $\mathbf{F}$ be an algebraic real vector bundle on $X$ with rank $\mathbf{F}=\operatorname{dim} X$. If $X$ has no compact connected components, then $\mathbf{F}$ has a one-dimensional trivial summand.

Proof. Let $d=\operatorname{dim} X$ and let $f: X \rightarrow \mathbb{G}_{d}\left(\mathbb{R}^{n}\right)$ be a regular map with $\mathbf{F} \cong$ $f^{*} \Gamma_{d}\left(\mathbb{R}^{n}\right)$, where $\mathbb{G}_{d}\left(\mathbb{R}^{n}\right)$ is the Grassmannian of $d$-dimensional vector subspaces of $\mathbb{R}^{n}$ and $\Gamma_{d}\left(\mathbb{R}^{n}\right)$ is the tautological vector bundle on $\mathbb{G}_{d}\left(\mathbb{R}^{n}\right)$. By Hironaka's theorem on resolutions of singularities, we may assume that $X$ is an open subvariety of a compact nonsingular variety $\bar{X}$. We regard $f: \bar{X}-\rightarrow \mathbb{G}_{d}\left(\mathbb{R}^{n}\right)$ as a rational map. According to Hironaka's theorem on resolution of points of indeterminacy, there exists a regular map $\pi: X^{\prime} \rightarrow \bar{X}$ such that $\pi$ is the composite of finitely many blowups with nonsingular centers, $\pi$ induces a biregular isomorphism between $\pi^{-1}(X)$ and $X$, and the rational map $f \circ \pi: X^{\prime} \rightarrow \mathbb{G}_{d}\left(\mathbb{R}^{n}\right)$ is actually regular. In particular, $\mathbf{F}^{\prime}:=(f \circ \pi)^{*} \Gamma_{d}\left(\mathbb{R}^{n}\right)$ is an algebraic vector bundle on $X^{\prime}$. In order to simplify notation, we identify $X$ with $\pi^{-1}(X)$. Then $\mathbf{F}_{\mid X}^{\prime}=\mathbf{F}$. It suffices to find an algebraic section $s: X^{\prime} \rightarrow \mathbf{F}^{\prime}$ with $Z(s) \subset X^{\prime} \backslash X$, where $Z(s)=\left\{x \in X^{\prime} \mid s(x)=\right.$ $0\}$.

Case 1. Suppose that $\operatorname{dim} X \geq 2$. We may assume that for each connected component $C$ of $X^{\prime}$, the set $C \backslash X$ is infinite (if necessary, blow up $X^{\prime}$ at a point in $C \backslash X)$. Let $u: X^{\prime} \rightarrow \mathbf{F}^{\prime}$ be a $\mathcal{C}^{\infty}$ section transverse to the zero section. Then the zero locus $Z(u)$ of $u$ is a finite set. Clearly, there exists a $\mathcal{C}^{\infty}$ diffeomorphism $h: X^{\prime} \rightarrow X^{\prime}$, homotopic to the identity map, such that $h^{-1}(Z(u)) \subset X^{\prime} \backslash X$. The pullback section $h^{*} u: X^{\prime} \rightarrow h^{*} \mathbf{F}^{\prime}$ is transverse to the zero section and $Z\left(h^{*} u\right)=h^{-1}(Z(u))$. Since $h$ is homotopic to $i d_{X}$, the vector bundles $h^{*} \mathbf{F}^{\prime}$ and $\mathbf{F}^{\prime}$ $\operatorname{are} \mathcal{C}^{\infty}$ isomorphic. Consequently, we can find a smooth section $v: X^{\prime} \rightarrow \mathbf{F}^{\prime}$ which is transverse to the zero section and satisfies $Z(v) \subset X^{\prime} \backslash X$. By [1, pp. 309, 321], $v$ can be approximated in the $\mathcal{C}^{\infty}$ topology by an algebraic section $s: X^{\prime} \rightarrow \mathbf{F}^{\prime}$ with $s_{\mid Z(v)}=0$. If $s$ is sufficiently close to $v$, then $Z(s)=Z(v) \subset X^{\prime} \backslash X$.

Case 2. Suppose that $\operatorname{dim} X=1$. Each connected component of $X^{\prime}$ is diffeomorphic to a circle. Thus there exists a smooth section $v: X^{\prime} \rightarrow \mathbf{F}^{\prime}$ which is transverse to the zero section and satisfies $Z(v) \subset X^{\prime} \backslash X$. Now we get $s$ as in Case 1.

Proof of Theorem 1.1. Point (i) follows from Theorem 2.2 and Lemma 2.3, whereas (ii) is a consequence of Theorem 2.2 and Lemma 2.4.

Proof of Theorem 1.2. If $Y^{r}$ is orientable (as $\mathcal{C}^{\infty}$ manifold), then the normal bundle of $Y^{r}$ in $R^{2 r}$ has a nowhere zero $\mathcal{C}^{\infty}$ section [11], and hence in view of [1, p.309], it has an algebraic one-dimensional summand. Consequently, $Y^{r}$ is contained in a nonsingular hypersurface in $\mathbb{R}^{2 r}$.

The orientability of $Y^{r}$ is essential here. Indeed, we have: 
Example 2.5. Let $f: \mathbb{P}^{2}(\mathbb{R}) \rightarrow \mathbb{R}^{4}$ be an algebraic embedding given by the formula

$$
f\left(\left(x_{1}: x_{2}: x_{3}\right)\right)=\frac{1}{x_{1}^{2}+2 x_{2}^{2}+3 x_{3}^{2}}\left(x_{1}^{2}+x_{2}^{2}+x_{3}^{2}, x_{1} x_{2}, x_{1} x_{3}, x_{2} x_{3}\right) .
$$

It is easy to see that $Y=f\left(\mathbb{P}^{2}(\mathbb{R})\right)$ is a nonsingular affine variety. According to [11], the normal bundle of any embedding of $\mathbb{P}^{2}(\mathbb{R})$ in $\mathbb{R}^{4}$ does not have a nowhere vanishing $\mathcal{C}^{\infty}$ section. According to Theorem 2.2, $Y$ is not contained in any nonsingular hypersurface in $\mathbb{R}^{4}$.

In the general case the orientability of $Y$ does not help. Let $X^{2 m}$ be a nonsingular variety and let $Y^{m}$ be a nonsingular orientable subvariety of $X^{2 m}$. We show that in general there does not exist a nonsingular hypersurface $H \subset X^{2 m}$, such that $Y^{n} \subset H$. Indeed we have:

Example 2.6. Let $m$ be an even number and let $\mathcal{S}^{m}$ be an $m$-dimensional sphere. Let $\mathbf{F}=T \mathcal{S}^{m}$ be the tangent bundle of the sphere $\mathcal{S}^{n}$. Now let $X^{2 m}$ denote the total space of this vector bundle. Then $\mathcal{S}^{m} \subset X^{2 m}$ (as the zero-section) and $\mathbf{N}_{X^{2 m}} / \mathcal{S}^{m} \cong \mathbf{F}$. It is well-known that the bundle $\mathbf{F}$ does not have a one-dimensional trivial summand. In particular $\mathcal{S}^{m}$ is not contained in any nonsingular hypersurface in $X^{2 m}$ (see Theorem 2.2).

\section{Holomorphic case}

Now we prove a similar result in the holomorphic setting. Let $X$ be a Stein manifold with the sheaf $\mathcal{O}$ of holomorphic functions. If $Y$ is a Stein submanifold of $X$, then the ideal $I(Y)=\left\{f \in \mathcal{O}(X): f_{\mid Y}=0\right\}$ is finitely generated ( see [3], Theorem 7.5.4). If $I(Y)$ is principal we say that $Y$ is a hypersurface. For Stein manifolds, Lemma 2.1 takes the following form:

Lemma 3.1. Let $Y \subset X$ be Stein manifolds and let $s, g_{1}, \ldots, g_{r}$ be holomorphic functions on $X$ such that $d_{y} s \neq 0$ for every $y \in Y$ and $I(Y)=\left(g_{1}, \ldots, g_{r}\right)$. Then the hypersurface $V\left(s+\sum_{i=1}^{r} \beta_{i} g_{i}^{2}\right)$ in $X$ is nonsingular for generic $\beta_{i} \in \mathbb{C}$.

Proof. Let $f: X \rightarrow \mathbb{C}$ be a holomorphic function. Then $0 \in \mathbb{C}$ is a regular value of $f$ if and only if $0 \in \mathbb{R}^{2} \cong \mathbb{C}$ is a regular value of $f$ regarded as real $\mathcal{C}^{\infty}$ map. This allows us to conclude arguing as in the proof of Lemma 2.1.

We have the following counterpart of Theorem 2.2, with a completely analogous proof:

Theorem 3.2. Let $Y \subset X$ be Stein manifolds. Then $Y$ is contained in some nonsingular Stein hypersurface $V(f) \subset X$ if and only if the normal bundle of $Y$ contains a one-dimensional trivial summand i.e.,

$$
\mathbf{N}_{X / Y}=\mathbf{T} \oplus \mathbf{E}^{1}
$$

where $\mathbf{E}^{1}$ denotes a trivial line bundle. 
It is well-known that an $n$-dimensional Stein manifold has the homotopy type of a (real) $n$-dimensional CW complex ( see [8]). Complex vector bundles on such $\mathrm{CW}$ complexes have the following nice property:

Theorem 3.3. ([6], p. 111) Let $Y$ be an $r$-dimensional $C W$ complex and let $\mathbf{F}$ be a complex vector bundle on $Y$ of rank $k$. If $r \leq 2 k-1$, then $\mathbf{F}$ has a one-dimensional trivial summand.

Proof of Theorem 1.3. We will make use of Grauert's theorem on the Oka principle for vector bundles which says that on Stein spaces the holomorphic and topological classifications coincide (see for example [2]). Therefore we can use the topological theory of complex vector bundles. Moreover, since every $n$-dimensional Stein manifold has a homotopy type of a (real) $n$-dimensional CW complex, if we study vector bundles on $X$, we can assume that $X$ itself is a $n$-dimensional $\mathrm{CW}$ complex.

The normal bundle $\mathbf{F}=\mathbf{N}_{X / Y}$ has rank $n-r$. By Theorem 3.3 it has a trivial one-dimensional summand if $r \leq 2(n-r)-1$, i.e., if $(3 r+1) / 2 \leq n$.

Now we show that the assumption $(3 r+1) / 2 \leq n$ is sharp.

Example 3.4. Consider the variety $\Gamma^{5}=\left\{(x, y) \in \mathbb{C}^{3} \times \mathbb{C}^{3}: \sum_{i=1}^{3} x_{i} y_{i}=1\right\}$. By the Raynaud Theorem (see [9]) the holomorphic vector bundle $\mathbf{F}$ on $\Gamma$ given by the unimodular row $\left(x_{1}, x_{2}, x_{3}\right)$ is not free. Since every stably trivial line bundle is trivial and rank $\mathbf{F}=2$, we see that the vector bundle $\mathbf{F}$ does not have a onedimensional trivial summand.

Now let $X^{7}$ denote the total space of this vector bundle. Then $\Gamma \subset X$ (as the zero-section) and $\mathbf{N}_{X / \Gamma} \cong \mathbf{F}$. As we observed the bundle $\mathbf{F}$ does not have a onedimensional trivial summand. In particular $\Gamma$ is not contained in any nonsingular hypersurface in $X$ (see Theorem 3.2). Hence the inequality $(3 r+1) / 2 \leq n$ cannot be replaced by the inequality $(3 r+1) / 2 \leq n+1$.

Open Access This article is distributed under the terms of the Creative Commons Attribution 4.0 International License (http://creativecommons.org/licenses/by/4.0/), which permits unrestricted use, distribution, and reproduction in any medium, provided you give appropriate credit to the original author(s) and the source, provide a link to the Creative Commons license, and indicate if changes were made.

\section{References}

[1] Bochnak, J., Coste, M., Roy, M.F.: Real Algebraic Geometry. Springer, Berlin (2005)

[2] Forster, O.: Topologische Methoden in der Theorie Steinischer Räume. Actes du Congres International des Mathematiciens, Nice, 1970, Tome 2, pp. 613-618, GauthiersVillars, Paris (1971)

[3] Forstneric, F.: Stein Manifolds and Holomorphic Mappings. Springer, Berlin (2011)

[4] Greco, S., Valabrega, P.: On the singular locus of a complete intersection through a variety in projective space. Bollettino U.M.I. VI-D 113-145 (1983) 
[5] Guillemin, V., Pollack, A.: Differential Topology. Prentice-Hall Inc., Englewood Cliffs (1974)

[6] Husemoller, D.: Fibre Bundles. Springer, New York (1994)

[7] Kleiman, S., Altman, A.: Bertini theorems for hypersurface sections containing a subscheme. Commun. Algebra 7(8), 775-790 (1979)

[8] Milnor, J.: Morse Theory, Annals of Mathematics Studies, vol. 51. Princeton University Press, Princeton (1963)

[9] Raynaud, M.: Modules projectifs universels. Invent. Math. 6, 1-26 (1968)

[10] Serre, J.P.: Modules projectifs et espaces fibres a fibre vectorielle. Sem. Dubreil-Pisot, No. 23, Paris (1957/1958)

[11] Whitney, H.: On the Topology of Differentiable Manifolds. Lectures in Topology. University of Michigan Press, pp. 101-141 (1941) 